\title{
Biologia reprodutiva de Tapirira guianensis Aubl. (Anacardiaceae), uma espécie dióica em mata de galeria do Triângulo Mineiro, Brasil.
}

\author{
EDDIE LENZA ${ }^{1,3}$ e PAULO EUGÊNIO OLIVEIRA ${ }^{2}$
}

(recebido: 8 de abril de 2004; aceito: 18 de novembro de 2004)

\begin{abstract}
Reproductive biology of Tapirira guianensis Aubl. (Anacardiaceae), a dioecious species, in a gallery forest of Triângulo Mineiro, Brazil). The reproductive biology of a Tapirira guianensis population was studied in Uberlândia, MG. Between September 1997 and April 2000 the phenology was observed for 17 individuals. Floral biology studies involved floral morphology and rewards, anthesis and flower longevity, inflorescence length and number of flowers, flower visitors and pollinators and breeding system. Tapirira guianensis presented massive annual flowering with quick and well synchronized blooming peaks of both male and female individuals. Flowers of both sexes are small (ca. $3 \mathrm{~mm}$ across) and inconspicuous. Male inflorescences are larger (Mann-Whitney, $U=1769,5 ; p<0,001$ ) and bear more flowers (Mann-Whitney, $U=3 ; p<0,001$ ) than the female ones. Male flower offer pollen and nectar while female flowers produce only nectar to flower visitors, all insects (41 species), mainly small social bees and flies. Flower opening occurred both during the day and night for flowers of both sexes and female flowers presented greater longevity. Fruit production and maturation after cross pollination (23,5\% and $12,1 \%$ respectively) were much larger than from apomixis experiments ( $1,3 \%$ and $0,45 \%$ respectively) $\left(\chi_{0,05,1}^{2}=561,4 ; p<0,001\right.$ for fruit production; $\chi_{0,05,1}^{2}=283,8 ; p<0,001$ for fruit maturation). Synchronous flower opening, greater floral display of male inflorescences and greater longevity of female flowers of $T$. guianensis probably increased pollination and consequently fruit production and maturation. Low fruit production from apomixis shows the vital role of pollination agents to the reproduction of $T$. guianensis, a species of widespread distribution and ecological importance.
\end{abstract}

Key words - Anacardiaceae, breeding systems, dioecy, phenology, pollination

RESUMO - (Biologia reprodutiva de Tapirira guianensis Aubl. (Anacardiaceae), uma espécie dióica, em mata de galeria do Triângulo Mineiro, Brasil). Foi estudada a biologia reprodutiva de uma população de Tapirira guianensis em Uberlândia, MG. Entre setembro de 1997 e abril de 2000 foi caracterizado o comportamento fenológico reprodutivo de 17 indivíduos da população. Nas análises da biologia floral foram determinados a morfologia e os recursos florais, o período de abertura e de duração das flores, o comprimento e o número de flores por inflorescências, os visitantes florais e o sistema reprodutivo. Tapirira guianensis apresentou floração anual e massiva com picos de floração rápidos e altamente sincrônicos entre os dois sexos. As flores dos dois sexos são pequenas (ca. $3 \mathrm{~mm}$ de diâmetro) e inconspícuas. As inflorescências masculinas são maiores (Mann-Whitney, $U=1769,5 ; p<0,001)$ e produzem mais flores (Mann-Whitney, $U=3 ; p<0,001)$ que as femininas. As flores masculinas oferecem pólen e néctar e as femininas apenas néctar aos visitantes florais, todos insetos (41 espécies), principalmente pequenas abelhas sociais e moscas. A antese foi tanto diurna quanto noturna para os dois tipos florais e as flores femininas apresentaram maior longevidade. A produção e maturação dos frutos por polinização cruzada $(23,5 \%$ e $12,1 \%$ respectivamente) foram maiores que aquelas observadas pelos testes de apomixia $\left(1,3 \%\right.$ e $0,45 \%$ respectivamente) $\left(\chi_{0,05,1}^{2}=561,4 ; p<0,001\right.$ para frutos produzidos; $\chi_{0,05,1}^{2}=283,8 ; p<0,001$ para frutos maturados). A abertura sincrônica das flores, o maior "display" floral de inflorescências masculinas e a maior longevidade das flores feminina em T. guianensis, provavelmente aumentaram os níveis de polinização e conseqüentemente a produção e maturação de frutos na espécie. A baixa formação de frutos por apomixia revela o papel vital dos agentes polinizadores para a reprodução de T. guianensis, uma espécie de ampla distribuição e importância ecológica.

Palavras-chave - Anacardiaceae, dioicia, fenologia, polinização, sistemas reprodutivos

\section{Introdução}

A presença de indivíduos masculinos e femininos dentro de populações de plantas caracteriza o sistema

1. Universidade de Brasília, Instituto de Ciências Biológicas, Departamento de Ecologia, 70910-900 Brasília, DF, Brasil.

2. Universidade Federal de Uberlândia, Instituto de Biologia, Caixa Postal 593, 38400-902 Uberlândia, MG, Brasil

3. Autor para correspondência: eddie@unb.br sexual conhecido por dioicia. Este sistema sexual ocorre em aproximadamente 6\% das angiospermas (Renner \& Ricklefs 1995) e possui ampla distribuição entre grupos filogeneticamente distintos (Bawa et al. 1985a, Bullock 1985).

Flores de espécies dióicas são geralmente pequenas e pouco especializadas (Bawa 1980a, Ibarra-Manríquez \& Oyama 1992, Oliveira 1996) possuindo cores claras e inconspícuas (Bawa 1980a, Givnish 1982, Sakai et al. 1995). Estudos com espécies dióicas em ecossistemas 
tropicais mostram forte associação entre a dioicia e a polinização por grande variedade de pequenos insetos generalistas (Bawa 1980a, Bawa et al. 1985b, Oliveira 1996), principalmente pequenas abelhas sociais generalistas e moscas (Bawa 1980a, Givnish 1982). Esses grupos de insetos são sensíveis a pequenas variações individuais na disponibilidade de recursos florais (Beach 1981). Em plantas hermafroditas, esse tipo de forrageamento promove o fluxo direcional de pólen de indivíduos com mais recursos para aqueles com menos recursos, sendo um dos mecanismos responsáveis pela evolução da dioicia (Beach 1981).

Em espécies dióicas, plantas masculinas produzem maior número de flores por inflorescência ou por indivíduo (Lloyd \& Webb 1977, Opler \& Bawa 1978, Bawa 1980b). Tal dimorfismo sexual é interpretado como uma das conseqüências da seleção sexual, que aumenta o "display" sexual de indivíduos masculinos e promove maior dispersão de pólen (Bawa \& Opler 1975, Bawa 1980b, Willson 1994).

A família Anacardiaceae ocorre principalmente em regiões tropicais e subtropicais (Heywood 1985), sendo predominantemente representada por espécies dióicas (Bullock 1985, Ibarra-Manríquez \& Oyama 1992, Renner \& Ricklefs 1995). Tapirira guianensis Aubl. é uma espécie dióica da família Anacardiaceae, amplamente distribuída em todo território brasileiro, principalmente em terrenos úmidos (Oliveira Filho \& Ratter 1995), sendo importante elemento do estrato lenhoso de matas do Brasil Central (Silva Júnior et al. 1998). Essa ampla distribuição contrasta com a presença de dioicia, que exige transferência de pólen entre indivíduos e está geralmente associada às plantas lenhosas mais raras e características de estágios tardios de sucessão (Ackerly et al. 1990, Ibarra-Manríquez \& Oyama 1992).

Este trabalho tem como objetivo analisar a biologia reprodutiva de uma população de Tapirira guianensis em mata de galeria no Município de Uberlândia, MG e avaliar se a eficiência do processo reprodutivo poderia ajudar a entender a razão da ampla distribuição e importância ecológica desta espécie.

\section{Material e métodos}

Este estudo foi desenvolvido no Parque do Sabiá, localizado no perímetro urbano da cidade de Uberlândia, MG (185' S e 48 $14^{\prime}$ 'W; altitude aproximada de $890 \mathrm{~m}$ ). O Parque do Sabiá possui remanescentes florestais de matas de galeria, mesófila e cerradão, que ocupam área aproximada de 30 hectares (Guilherme et al. 1998). O clima da região é do tipo Aw, de acordo com a classificação de Köppen, com duas estações bem definidas, um verão quente e chuvoso e um inverno frio e seco. A precipitação anual varia em torno de $1.550 \mathrm{~mm}$ e a temperatura média é de aproximadamente $22^{\circ} \mathrm{C}$ (Rosa et al. 1991). A temperatura e a pluviosidade média mensais do Parque do Sabiá durante o período de estudo estão apresentadas na figura 1.

Tapirira guianensis é uma espécie arbórea, perenifólia e com altura variando de 10 a 13 metros. As flores são unissexuais e estão dispostas em panículas axilares distais (Fleig 1989).

Em setembro de 1997 foram marcadas 27 plantas na população. O sexo dos indivíduos foi determinado no campo e no laboratório de Morfologia Vegetal da Universidade Federal de Uberlândia (UFU), onde foram analisadas 10 flores de cada indivíduo com auxílio de estereomicroscópio. Durante os períodos reprodutivos dos anos de 1998 e 1999, o sexo dos indivíduos foi novamente analisado, usando o mesmo procedimento, para verificar se ocorria mudança da expressão sexual entre estações reprodutivas consecutivas. Aplicou-se o teste de $\chi^{2}$ com correção Yates para continuidade (Zar 1999) para avaliar se a razão entre os dois sexos na população diferia significativamente da unidade nos três períodos reprodutivos estudados. Foi adotado o nível de significância de $5 \%$.

Doze indivíduos masculinos e sete femininos da população foram acompanhados entre setembro de 1997 e abril de 2000 para caracterização do comportamento fenológico reprodutivo. Durante o período reprodutivo do ano de 1997 os censos foram realizados em intervalos que variaram entre uma e duas semanas. No período reprodutivo de 1999 o intervalo entre os censos foi de uma semana. Durante a floração e a maturação dos frutos nestes dois períodos reprodutivos as observações foram realizadas em intervalos inferiores a uma semana. Os censos realizados durante o período reprodutivo do ano de 1998 tiveram

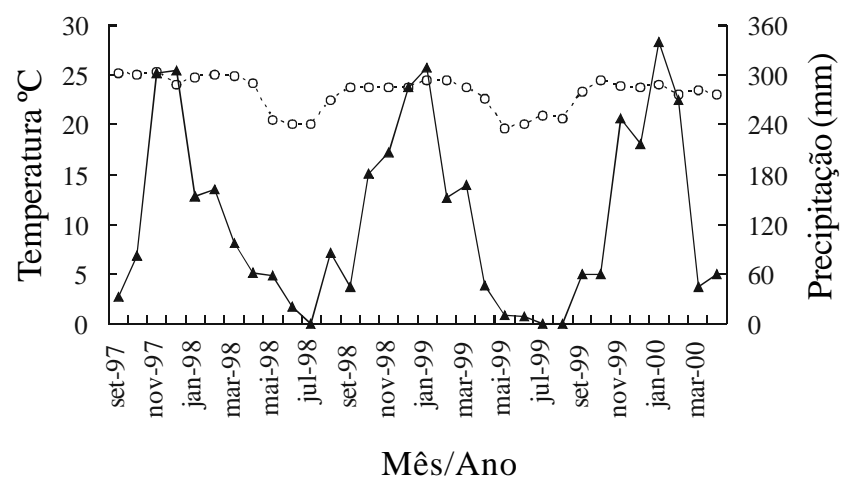

Figura 1. Climatograma do Parque do Sabiá, Uberlândia, MG durante o período de estudo. --O-- = Temperatura, $-\mathbf{\Lambda}-=$ Precipitação.

Figure 1. Climatic diagram of the Parque do Sabiá, Uberlândia, MG during the study period. --O-- = Temperature, $-\mathbf{-}-$ $=$ Precipitation. 
intervalos que variaram de uma semana a até mais de um mês.

Durante os censos foi determinada a presença de botões florais, flores, frutos imaturos e maduros. Foi realizada também uma análise fenológica quantitativa através de estimativas da apresentação ou "display" floral individual de acordo com o seguinte critério: 0 - indivíduo somente com botões florais; 1 - indivíduo com $1 \%$ a $10 \%$ de flores abertas; 2 - indivíduo com $11 \%$ a $60 \%$ de flores abertas; 3 - indivíduo com $61 \%$ a $100 \%$ de flores abertas. A intensidade de floração da população (IFP) foi determinada somando-se as estimativas individuais, para os 19 indivíduos, em cada um dos censos. Os picos de floração foram caracterizados quando $50 \%$ ou mais dos indivíduos da população possuíam flores. As análises fenológicas qualitativas e quantitativas para floração foram comparadas para avaliar se os picos de floração coincidiram com os maiores valores de IFP na população.

Em setembro de 1999 foi medido, com régua graduada em milímetros, o comprimento de 100 inflorescências em cinco indivíduos de cada sexo (20 inflorescências por indivíduo). As inflorescências foram coletadas de ramos escolhidos ao acaso nos indivíduos. De cada ramo foram coletadas, do ápice para a base, as inflorescências que apresentavam flores abertas.

Em outubro de 1999, foi determinado o número de flores por inflorescências para 10 inflorescências, escolhidas ao acaso, em cinco indivíduos de cada sexo (duas inflorescências por indivíduo). O único critério para a escolha das inflorescências foi que estas fossem coletadas de indivíduos que apresentassem mais de $60 \%$ das flores abertas, ou seja, categoria três de "display" floral.

As comparações entre os dois sexos quanto ao comprimento das inflorescências e ao número de flores por inflorescências foram realizadas através do teste de MannWhitney, adotando-se um nível de significância de 5\%.

A morfologia floral foi determinada a partir de análises de 40 flores de cada sexo (10 flores de quatro indivíduos) sob estereomicroscópio. Foram determinados o diâmetro das flores, o número, a disposição e a cor de cada um dos verticilos florais.

As duas formas florais foram analisadas quanto à presença e viabilidade dos grãos de pólen com o uso de carmim acético (Radford et al. 1974). A presença de néctar foi determinada, com uso de estereomicroscópio. Para as análises de pólen e néctar foram usadas 20 flores de cada sexo, ensacadas antes da antese, durante a floração de 1999. Adicionalmente, a presença de néctar foi determinada através de observações diretas das flores no campo, com o uso de lupa de bolso com aumento de cinco vezes.

Para a determinação do período de antese foram usadas quatro inflorescências em dois indivíduos distintos de cada sexo. As inflorescências foram marcadas antes que apresentassem flores abertas. A contagem das flores abertas foi feita em intervalos de duas horas entre 6:00 h e 18:00 h e em um único intervalo de 12 horas entre 18:00 h e 6:00 h. Este experimento teve duração de três dias para os indivíduos femininos (entre 18:00 h de 2/08/1999 e 18:00 h de 5/08/1999) e de quatro dias para os indivíduos masculinos (entre 18:00 h de 8/08/1999 e 18:00 h de 12/08/1999). As flores foram consideradas abertas quando os verticilos reprodutivos (anteras e estigmas) estavam expostos, mesmo que as pétalas não estivessem completamente distendidas. Vinte flores de cada sexo, neste estágio de desenvolvimento, foram analisadas quanto à sua receptividade estigmática pelo método de $\mathrm{H}_{2} \mathrm{O}_{2}$ a 3\% (Kearns \& Inouye 1993), a liberação de pólen e a presença de néctar.

Em agosto de 1999, a duração das flores foi determinada em quatro inflorescências masculinas e quatro femininas de dois indivíduos de cada sexo (duas inflorescências por indivíduo). Inicialmente foi determinado o período de antese. Posteriormente, as flores foram acompanhadas em intervalos aproximados de 12 horas (sempre às 8:00 h e às 18:00 h) por oito dias para as flores femininas e por sete dias para as flores masculinas. As flores femininas foram ensacadas antes da antese para evitar sua polinização.

Os visitantes florais foram capturados em três dias consecutivos de coleta durante a floração de 1997, entre 8:00 h e 12:00 h. As identificações foram realizadas por comparações com exemplares do Museu de História Natural da Universidade Federal de Uberlândia e, sempre que possível, confirmadas por especialistas. O comprimento do corpo dos visitantes florais foi medido e as espécies foram classificadas como pequenas $(\leq 12 \mathrm{~mm})$ ou grandes $(>12 \mathrm{~mm})$ (adaptado de Oliveira \& Gibbs 2000).

Para caracterização dos sistemas reprodutivos foram analisadas a produção e a maturação de frutos por polinização natural (2.835 botões) e por apomixia (2.422). Para cada um dos dois tratamentos foram escolhidos quatro indivíduos femininos e contados os botões florais de três inflorescências por indivíduo. No tratamento de polinização natural as flores ficaram expostas aos agentes polinizadores enquanto no tratamento de apomixia as inflorescências foram isoladas com sacos de organza para evitar que as flores fossem polinizadas. Os frutos formados foram acompanhados, em intervalos variáveis, desde o início de seu desenvolvimento até sua maturação (entre setembro de 1997 e janeiro de 1998). Foi aplicado o teste de $\chi^{2}$, com nível de significância de 5\%, para comparar a produção e maturação dos frutos entre os dois tratamentos.

\section{Resultados}

Dos 27 indivíduos marcados em 1997 (15 masculinos e 12 femininos) oito não floresceram em 1998 e dois em 1999. Nenhum indivíduo mudou de sexo entre os três períodos reprodutivos analisados. A razão entre indivíduos masculinos e femininos na população estudada foi de $1,25,1,11$ e 1,5 para os períodos reprodutivos de 1997, 1998 e 1999, respectivamente. Apesar da tendência de domínio de indivíduos masculinos 
nos três períodos reprodutivos, em nenhum deles houve diferença significativa na freqüência dos dois sexos $\left(1997-\chi_{0,05,1}^{2}=0,148 ; p=0,7003 ; 1998-\chi_{0,05,1}^{2}=0\right.$; $\left.p=1 ; 1999-\chi_{0,05,1}^{2}=0,64 ; p=0,4237\right)$.

A população de T. guianensis apresentou floração anual com picos ou pulsos de floração rápidos e altamente sincrônicos nos três períodos reprodutivos, tanto em nível populacional quanto entre plantas dos dois sexos (figura 2a, b, c). Três indivíduos, um masculino e dois femininos, floresceram entre abril e junho de 1999 (figura 2b), entretanto apresentaram inflorescências isoladas, sem que houvesse maturação de frutos. Por essa razão, esses três indivíduos não foram incluídos nas análises fenológicas.

No período reprodutivo de 1997 foram observados dois picos de floração (com $\geq 50 \%$ dos indivíduos com flor), com um intervalo aproximado de 20 dias entre eles, período no qual todos os indivíduos da população se mantiveram sem flores (figura 2a). Em 1998 foi observado apenas um pico de floração em outubro (figura 2b). Padrão fenológico de floração semelhante ao de 1997 foi observado durante a floração de 1999, entretanto foram observados cinco picos de floração (figura 2c). A floração de indivíduos no mês de dezembro, tanto em 1997 quanto em 1999 (figura 2a, c), não foi suficiente para a caracterização de mais um pico de floração. Neste período, os indivíduos possuíam número reduzido de flores abertas em inflorescências isoladas na planta, caracterizando o fim da estação reprodutiva. Durante a floração do ano de 1997 todos os 19 indivíduos da população floresceram nos dois picos de floração observados. Durante o período reprodutivo de 1999 todos os indivíduos floresceram e apenas três indivíduos masculinos e um feminino não floresceram em algum dos cinco picos de floração.

As análises quantitativas do comportamento fenológico da população de $T$. guianensis, mostraram que durante os picos de floração os indivíduos permaneceram com mais de $60 \%$ das flores abertas por um tempo normalmente inferior a uma semana. A abertura em massa das flores e a alta sincronia de floração entre os indivíduos contribuíram para os altos valores de intensidade de floração na população (IFP), observados nos picos de floração (figura $3 \mathrm{a}, \mathrm{b}, \mathrm{c}$ ).

O período entre os picos de floração para os anos de 1997 e 1999 foi marcado por nenhum ou poucos indivíduos com flores, geralmente com um "display" floral individual baixo, ou seja, com poucas flores abertas por indivíduo. Estes dois fenômenos são responsáveis pelos valores nulos ou muito baixos de IFP observados entre os picos de floração (figura 3a, c).
O desenvolvimento dos frutos no período reprodutivo de 1997 teve início em outubro e a maturação ocorreu entre os meses de janeiro e março de 1998. Para o período reprodutivo de 1999 a produção de frutos iniciou-se em agosto do mesmo ano, com maturação entre dezembro de 1999 e março de 2000. Nestes dois períodos os sete indivíduos femininos acompanhados produziram e maturaram frutos. No entanto, a produção e a maturação de frutos para o
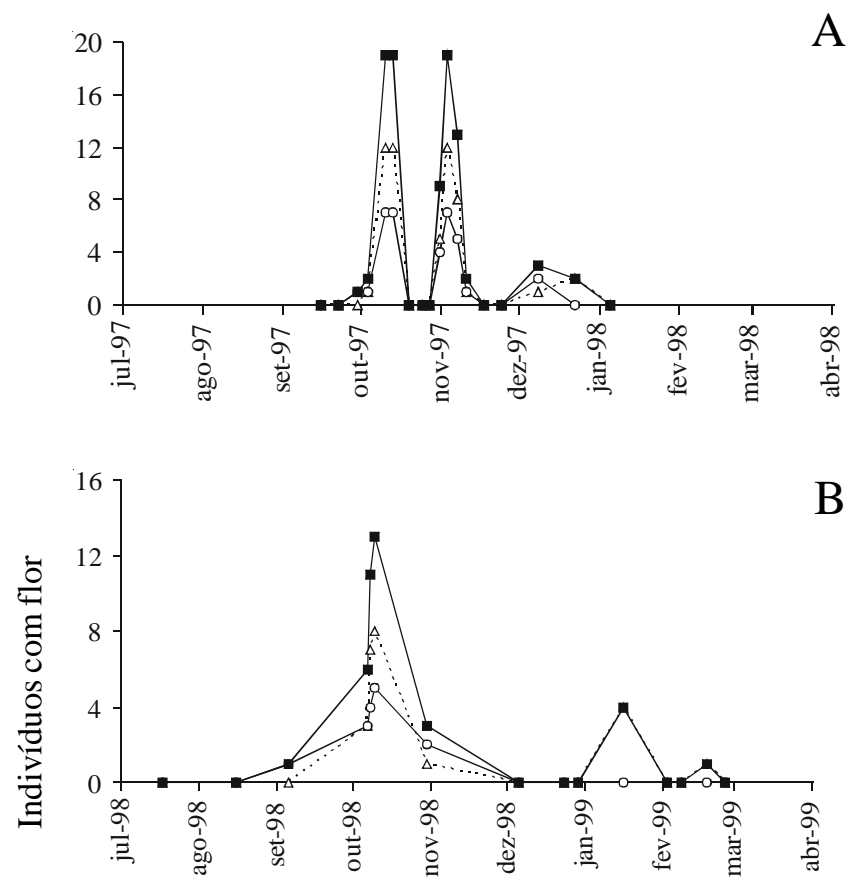

B

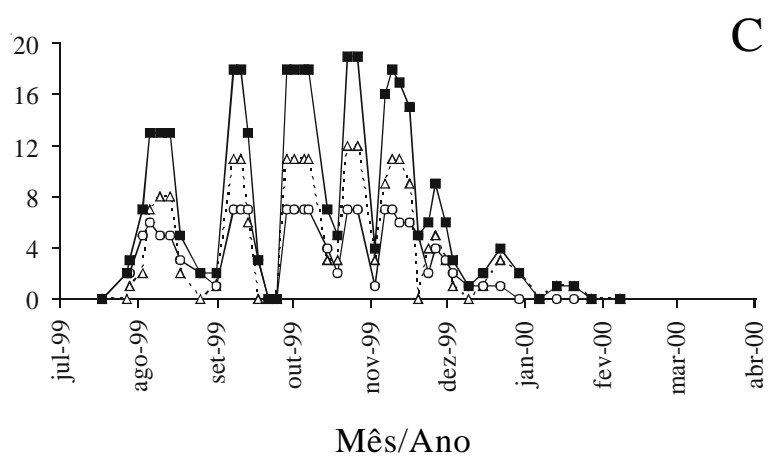

Figura 2. Fenologia de floração de Tapirira guianensis em mata de galeria do Parque do Sabiá, Uberlândia, MG. A. Período reprodutivo de 1997; B. Período reprodutivo de 1998; C. Período reprodutivo de 1999. -- $\triangle--=$ Masculino, $-0-=$ Feminino, $-\mathbf{-}-=$ População.

Figure 2. Flowering phenology of Tapirira guianensis in a gallery forest of the Parque do Sabiá, Uberlândia, MG. A. Reproductive period of 1997; B. Reproductive period of 1998; C. Reproductive period of 1999. -- $\triangle--=$ Male, $-0-=$ Female, $-\mathbf{-}=$ Population. 
período reprodutivo de 1998 foram muito baixas. Nesse período apenas quatro indivíduos produziram frutos e, destes, apenas dois foram observados com poucos frutos maduros nos meses de fevereiro e março de 1999.

O comprimento médio das inflorescências masculinas ( $\mathrm{X}=13,5 \mathrm{~cm} \pm 4,2 ; \mathrm{n}=100)$ foi significativamente maior que o das inflorescências femininas $(X=9 \mathrm{~cm} \pm 2,5 ; \mathrm{n}=100)$ (Mann-Whitney, $U=1769,5 ; p<0,001)$. O número médio de flores masculinas por inflorescência $(514 \pm 227 ; n=10)$ também foi significativamente maior que o de flores femininas (118 $\pm 44 ; \mathrm{n}=10)$ (Mann-Whitney, $U=3 ; p<0,001)$.

As flores dos dois sexos são pequenas (ca. $3 \mathrm{~mm}$ de diâmetro), pedunculadas, actinomorfas, pentâmeras, dialipétalas e gamossépalas, com ambos os verticilos esverdeados. Os estames, cinco alternipétalos e cinco alternissépalos, são livres e isodínamos (figura 4a, b, c), com anteras apresentando deiscência longitudinal.

Nas flores masculinas, os estames estão direcionados para cima e lateralmente (figura 4a, d), com anteras amarelas. $\mathrm{O}$ gineceu é atrofiado, piloso e não funcional (figura 4a), às vezes apresentando estigmas vestigiais. Nas flores femininas o gineceu é amarelo claro, globoso e bem desenvolvido (figura 4b), com ovário unilocular súpero. Os cinco estigmas são curtos e possuem superfície estigmática globosa (figura 4b). Os estames são menores e laterais ao ovário (figura 4b, 4e), possuindo anteras brancas sem grãos de pólen em seu interior. As flores masculinas oferecem néctar e pólen aos visitantes, enquanto as flores femininas possuem apenas néctar como recompensa. Ocasionalmente, foram observadas algumas flores hermafroditas em dois indivíduos femininos da população, que forneciam pólen e néctar. $\mathrm{O}$ gineceu destas flores possuía um óvulo, como nas flores femininas, no entanto o ovário era menos desenvolvido (figura 4c). $\mathrm{O}$ androceu era similar ao das flores masculinas e, apesar das anteras serem um pouco menores (figura 4c, f). Foi observada liberação de grãos de pólen bem desenvolvidos, entretanto a viabilidade desses não foi testada.

O número e percentagem de flores abertas durante os diferentes intervalos de observação estão apresentados na tabela 1. Houve abertura das flores femininas em todos os intervalos e apenas entre 12:00 h e 14:00 h não foi registrada antese de flores masculinas. A proporção de flores masculinas abertas entre 18:00 h e 6:00 h foi quase três vezes maior que a de femininas abertas no mesmo intervalo (tabela 1 ).

Das 60 flores parcialmente abertas analisadas, 20 de cada forma floral, apenas uma flor feminina e nenhuma flor masculina ou hermafrodita apresentaram néctar. Para as 20 flores masculinas e hermafroditas analisadas, 18 e sete, respectivamente, apresentaram anteras abertas com liberação de pólen. Os estigmas de todas as flores femininas e de 16 flores hermafroditas parcialmente abertas já estavam receptivos.
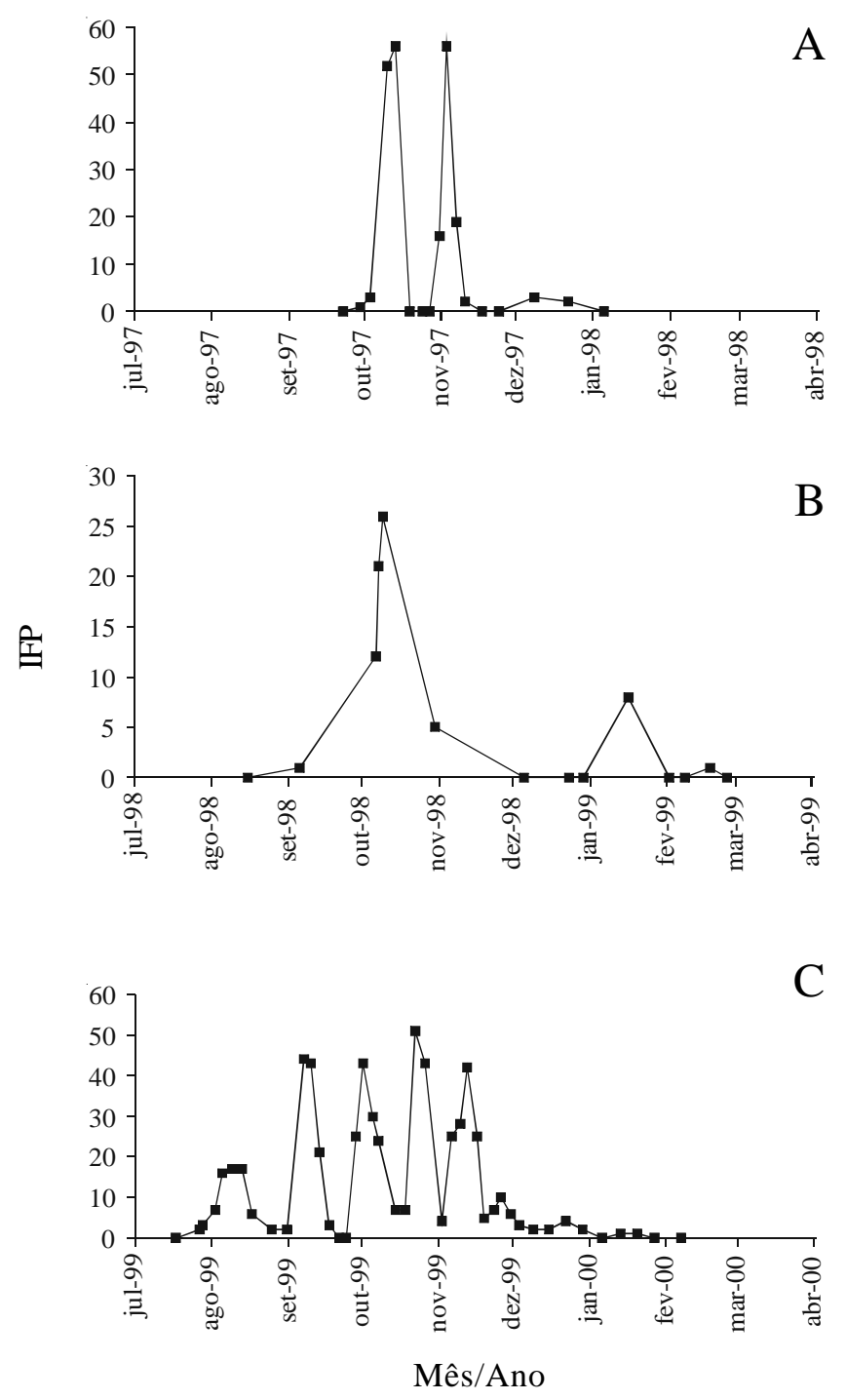

Figura 3. Intensidade de floração na população (IFP) de Tapirira guianensis em mata de galeria do Parque do Sabiá, Uberlândia, MG. IFPé a soma das estimativas de intensidade de floração nos indivíduos da população portando flores. A. Período reprodutivo de 1997. B. Período reprodutivo de 1998. C. Período reprodutivo de 1999.

Figure 3. Intensity of flowering in a population of Tapirira guianensis in a gallery forest of the Parque do Sabiá, Uberlândia, MG. IFP is the sum of flowering intensity estimates for the individuals bearing flowers. A. Reproductive period of 1997. B. Reproductive period of 1998. C. Reproductive period of 1999. 

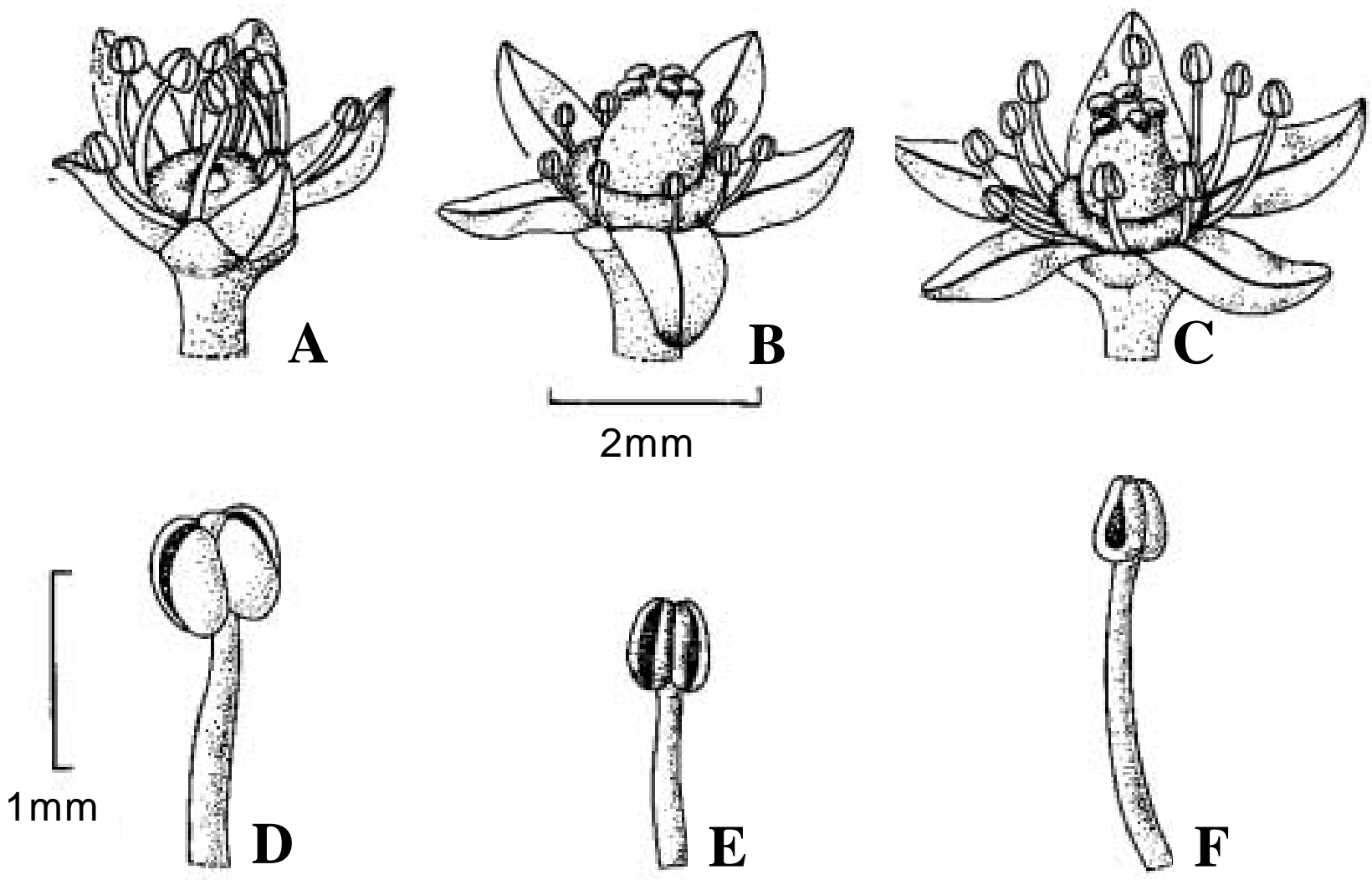

Figura 4. Morfologia floral de Tapirira guianensis em mata de galeria do Parque do Sabiá, Uberlândia, MG. A. Flor masculina; B. Flor feminina. C. Flor hermafrodita. D. Estame flor masculina. E. Estame flor feminina. F. Estame flor hermafrodita.

Figure 4. Floral morphology of Tapirira guianensis in a gallery forest of the Parque do Sabiá, Uberlândia, MG. A. Male flower. B. Female flower. C. Hermaphrodite flower. D. Stamen of male flower. E. Stamen of female flower. F. Stamen of hermaphrodite flower.

As flores femininas apresentaram maior longevidade quando comparadas às masculinas (tabela 2). Nenhuma flor feminina sofreu abscisão até

Tabela 1. Período de abertura de flores de Tapirira guianensis em mata de galeria do Parque do Sabiá, Uberlândia, MG.

Table 1. Tapirira guianensis flower opening period in a gallery forest of the Parque do Sabiá, Uberlândia, MG.

\begin{tabular}{lcc}
\hline Período & $0^{*} \mathrm{n}(\%)$ & क $\mathrm{n}(\%)$ \\
\hline 18:00 h-6:00 h & $52(58,4)$ & $26(20,8)$ \\
6:00 h-8:00 h & $9(10,1)$ & $13(10,4)$ \\
8:00 h - 10:00 h & $5(5,6)$ & $22(17,6)$ \\
10:00 h-12:00 h & $3(3,4)$ & $22(17,6)$ \\
12:00 h-14:00 h & $0(0)$ & $10(8,0)$ \\
14:00 h-16:00 h & $8(9)$ & $12(9,6)$ \\
16:00 h- 18:00 h & $12(13,5)$ & $20(16)$ \\
Total & 89 & 125 \\
\hline
\end{tabular}

Tabela 2. Longevidade de flores de Tapirira guianensis em mata de galeria no Parque do Sabiá, Uberlândia, MG. $\mathrm{AB}=$ Abscisão $\mathrm{MU}=$ Flores murchas ou com sinais de murchamento, no = Não observado.

Table 2. Longevity of Tapirira guianensis flowers in a gallery forest of the Parque do Sabiá, Uberlândia, MG. AB = Abcision; $\mathrm{MU}=$ Wilting flowers; no $=$ No observation

\begin{tabular}{|c|c|c|c|c|}
\hline \multirow{2}{*}{$\begin{array}{l}\text { Período } \\
\text { (h) }\end{array}$} & \multicolumn{2}{|c|}{$0^{\top}(\mathrm{n}=37)$} & \multicolumn{2}{|c|}{ 우 $(\mathrm{n}=32)$} \\
\hline & $\mathrm{AB}$ & MU & $\mathrm{AB}$ & MU \\
\hline 24 & 6 & 1 & 0 & 0 \\
\hline 48 & 15 & 3 & 0 & 1 \\
\hline 72 & 19 & 3 & 0 & 1 \\
\hline 96 & 23 & 4 & 0 & 1 \\
\hline 120 & 24 & 7 & 0 & 3 \\
\hline 144 & 26 & 8 & 0 & 6 \\
\hline 168 & 27 & 7 & 0 & 12 \\
\hline 192 & no & no & 0 & 16 \\
\hline
\end{tabular}


Tabela 3. Visitantes florais de Tapirira guianensis em mata de galeria no Parque do Sabiá, Uberlândia, MG. Identificação, comprimento do corpo e número de coletas.

Table 3. Floral visitors of Tapirira guianensis in a gallery forest of the Parque do Sabiá, Uberlândia, MG. Identification, body length and number of collections.

\begin{tabular}{|c|c|c|}
\hline Ordem/Família/Espécie & Corpo (mm) & Coletas \\
\hline \multicolumn{3}{|l|}{ HYMENOPTERA } \\
\hline \multicolumn{3}{|l|}{ APIDAE } \\
\hline Apis mellifera & 10 & 3 \\
\hline Scaptotrigona cf. depilis & 6 & 4 \\
\hline Tetragonisca angustula & 4 & 3 \\
\hline Trigona sp. & 7 & 1 \\
\hline \multicolumn{3}{|l|}{ ANTHOPHORIDAE } \\
\hline Exomalopsis cf. analis & 7 & 1 \\
\hline \multicolumn{3}{|l|}{ HALICTIDAE } \\
\hline Augochloropsis sp. & 9 & 1 \\
\hline Temnosoma sp. & 10 & 1 \\
\hline Halictidae sp.1 & 4 & 1 \\
\hline Halictidae sp.2 & 6 & 1 \\
\hline Halictidae sp.3 & 7 & 3 \\
\hline Halictidae sp.4 & 5 & 3 \\
\hline \multicolumn{3}{|l|}{ POMPILIDAE } \\
\hline sp. & 12 & 1 \\
\hline \multicolumn{3}{|l|}{ VESPIDAE } \\
\hline Brachygastra sp. & 8 & 1 \\
\hline Polybia flavifrons & 9 & 1 \\
\hline Polybia sp. & 10 & 1 \\
\hline \multicolumn{3}{|l|}{ SPHECIDAE } \\
\hline sp. & 9 & 2 \\
\hline \multicolumn{3}{|l|}{ FORMICIDAE } \\
\hline Camponotus sp. & 4 & 1 \\
\hline \multicolumn{3}{|l|}{ DIPTERA } \\
\hline \multicolumn{3}{|l|}{ CALIPHORIDAE } \\
\hline Chrysomia albiceps & 8 & 7 \\
\hline Chrysomia megacephala & 8 & 2 \\
\hline Phaenicia eximia & 7 & 3 \\
\hline \multicolumn{3}{|l|}{ SARCOPHAGIDAE } \\
\hline sp. & 7 & 1 \\
\hline \multicolumn{3}{|l|}{ SYRPHIDAE } \\
\hline Ornidia obesa & 11 & 5 \\
\hline Syrphidae sp.1 & 10 & 6 \\
\hline Syrphidae sp. 2 & 12 & 1 \\
\hline
\end{tabular}

oito dias (192 horas) após a antese, embora neste período 16 flores $(50 \%)$ já apresentassem sinais de murcha (tabela 2). Por outro lado, ao fim do terceiro dia (72 horas) mais da metade $(51 \%)$ das flores masculinas haviam caído, sendo que ao final do experimento, ou seja, sete dias (168 horas) após a antese, restaram apenas 10 flores (27\%), das quais sete estavam murchas ou com sinais de murchamento (tabela 2). As 10 flores

\begin{tabular}{|c|c|c|}
\hline Ordem/Família/Espécie & Corpo (mm) & Coletas \\
\hline Syrphidae sp.3 & 11 & 1 \\
\hline Syrphidae sp.4 & 9 & 1 \\
\hline Syrphidae sp.5 & 16 & 1 \\
\hline Syrphidae sp.6 & 13 & 1 \\
\hline Syrphidae sp.7 & 7 & 1 \\
\hline \multicolumn{3}{|l|}{ TABANIDAE } \\
\hline sp. & 7 & 1 \\
\hline \multicolumn{3}{|l|}{ TACHINIDAE } \\
\hline sp. & 14 & 3 \\
\hline \multicolumn{3}{|l|}{ COLEOPTERA } \\
\hline \multicolumn{3}{|l|}{ CHRYSOMELIDAE } \\
\hline sp. & 3 & 1 \\
\hline \multicolumn{3}{|l|}{ CURCULIONIDAE } \\
\hline sp. & 3 & 1 \\
\hline \multicolumn{3}{|l|}{ TENEBRIONIDAE } \\
\hline sp. & 6 & 4 \\
\hline \multicolumn{3}{|l|}{ HEMIPTERA } \\
\hline \multicolumn{3}{|l|}{ COREIDAE } \\
\hline sp. & 6 & 2 \\
\hline \multicolumn{3}{|l|}{ LIGAEIDAE } \\
\hline sp. & 10 & 1 \\
\hline \multicolumn{3}{|l|}{ NABIDAE } \\
\hline sp. & 5 & 2 \\
\hline \multicolumn{3}{|l|}{ PYRRHOCORIDAE } \\
\hline sp. & 8 & 3 \\
\hline \multicolumn{3}{|l|}{ LEPDOPTERA } \\
\hline \multicolumn{3}{|l|}{ NYNPHALIDAE } \\
\hline Methona themisto & 21 & 1 \\
\hline \multicolumn{3}{|l|}{ HOMOPTERA } \\
\hline \multicolumn{3}{|l|}{ CICADELLIDAE } \\
\hline sp. & 3 & 1 \\
\hline \multicolumn{3}{|l|}{ MEMBRACIDAE } \\
\hline sp. & 12 & 1 \\
\hline Total (Indivíduos) & & 80 \\
\hline Total (Espécies) & & 41 \\
\hline
\end{tabular}

masculinas coletadas no sétimo dia, apresentaram anteras brancas, acastanhadas, deiscentes e vazias. Nenhuma das flores possuía néctar. O não isolamento destas flores dificulta qualquer tipo de análise a respeito da sua funcionalidade, uma vez que os recursos podem ter sido consumidos pelos visitantes florais. Foi possível detectar apenas a alteração na cor dos estames, que pode ter comprometido o reconhecimento das flores 
pelos visitantes florais, uma vez que não foram observadas visitas a estas flores.

As flores femininas e hermafroditas do oitavo dia foram analisadas separadamente quanto à presença de recursos florais. Estas análises só foram possíveis porque as flores ficaram ensacadas ao longo do experimento, o que impediu a retirada de recursos pelos visitantes. Das 19 flores femininas 17 ainda apresentavam néctar. Das 13 flores hermafroditas todas possuíam anteras deiscentes expondo grãos de pólen amarelos e 12 apresentaram néctar. Sendo assim, as flores femininas e hermafroditas no oitavo dia após a antese ainda apresentam recursos capazes de atrair os visitantes florais.

Foram coletados 80 visitantes florais, todos da classe Insecta, pertencentes a seis ordens, 22 famílias e 41 espécies (tabela 3 ). As ordens de visitantes florais com maior número de espécies foram Hymenoptera e Diptera com 17 e 14 espécies, respectivamente, que somadas representaram $75,6 \%$ do total de espécies coletadas. Trinta e sete $(90 \%)$ espécies enquadraramse na categoria "pequena" e quatro $(10 \%)$ na "grande". Dentro do grupo das abelhas, ou seja, as ordens Apidae, Anthophoridae e Halictidae, todas as espécies foram "pequenas" (tabela 3 ).

$\mathrm{Na}$ análise do sistema reprodutivo, a produção de frutos por polinização natural $(23,5 \%)$ foi significativamente maior do que por apomixia $(1,3 \%)$ $\left(\chi_{0,05,1}^{2}=561,4 ; p<0,001\right)$. Da mesma forma, a maturação de frutos obtidos por polinização natural $(12,1 \%)$ foi significativamente maior do que a maturação por apomixia $(0,45)\left(\chi_{0,05,1}^{2}=283,8 ; p<0,001\right)$. Entretanto, os frutos apomíticos que maturaram desenvolveram-se normalmente.

\section{Discussão}

A população de T. guianensis apresentou floração anual do tipo "pulsed bang" ou floração em pulsos (Oliveira et al.1991, Proença \& Gibbs 1994). Esse tipo de floração, em picos rápidos e sincrônicos entre os indivíduos da população, termina por atrair polinizadores oportunistas, como moscas e ou com capacidade de recrutamento, como abelhas sociais (Newstrom et al. 1994), que são os principais visitantes florais de T. guianensis. Certamente, os intervalos de observação adotados durante o período reprodutivo de 1998, algumas vezes superiores a um mês, foram inadequados para detectar eventos rápidos de floração, como aqueles observados em 1997 e 1999, caso estes tenham ocorrido. Os episódios de floração também variaram de ano para ano em Guarea rhopalocarpa Radlk., uma Meliaceae dióica da Costa Rica (Bullock et al. 1983). Entretanto, ao contrário de $T$. guianensis, naquela espécie os episódios envolveram proporções diferentes de indivíduos de cada sexo, ocorreram de modo intermitente ao longo do ano e nunca envolveram eventos massivos de floração como os observados em T. guianensis.

Indivíduos florescendo sincronicamente em uma população atraem maior número de visitantes florais (Augspurger 1981), aumentam as taxas de visitas às flores e o transporte de pólen entre plantas (Augspurger 1980, Primack 1980) e, conseqüentemente, elevam os níveis de polinização (Augspurger 1981). Adicionalmente, em espécies dióicas, como é o caso de T. guianensis, a sincronia de floração entre indivíduos dos dois sexos pode também ter papel importante no que diz respeito à polinização, pois, como os picos de floração são breves, o fluxo de pólen entre os indivíduos poderia ser comprometido caso houvesse uma grande separação temporal na apresentação das flores pelos dois sexos.

A abertura sincrônica das flores também é mais importante para aumentar o "display" floral individual do que o número total de flores por plantas (Muenchow \& Delesalle 1994). As flores de T. guianensis, pouco conspícuas e com pouco ou nenhum odor, provavelmente seriam detectadas com mais dificuldade pelos agentes polinizadores caso a floração não ocorresse em picos. Assim, as centenas de flores fazem com que as inflorescências funcionem como unidades de atração para os visitantes florais (Croat 1979).

Por outro lado, o grande número de flores apresentadas por um indivíduo em curto espaço de tempo, pode restringir o movimento dos polinizadores e, conseqüentemente, o fluxo de pólen entre plantas da população, favorecendo a autopolinização em espécies hermafroditas (Bawa \& Opler 1975, Stephenson 1982). Mas mesmo em plantas dióicas, como é o caso de T. guianensis, a floração massiva e a restrição dos movimentos entre plantas, pode limitar a fertilização das flores femininas. Nesses casos, pequenas diferenças temporais (de até um dia) na apresentação das flores entre os indivíduos, uma certa assincronia, podem promover o fluxo de pólen (Stephenson 1982).

Flores pequenas e inconspícuas e pouco especializadas, associadas ao dimorfismo sexual pouco acentuado, como em T. guianensis, são características muito comuns em espécies dióicas tropicais (Bawa \& Opler 1975, Bawa 1980a, Oliveira 1996). A semelhança entre flores masculinas e femininas em espécies dióicas facilita seu reconhecimento pelos agentes polinizadores 
e permite que ocorra o fluxo de pólen entre os sexos (Bawa 1980b, Armstrong \& Irvine 1989b, Freeman et al. 1997). Apesar da separação funcional entre os sexos das flores de T. guianensis, há manutenção de estames nas flores femininas. Órgãos rudimentares não funcionais são relativamente comuns em flores de espécies dióicas (Bawa \& Opler 1975, Fleig 1989, Kato \& Nagamasu 1995). A presença de estames não funcionais em flores femininas pode ter, ainda, a função de atrair visitantes florais que buscam pólen como recompensa (Bawa 1977, Bawa 1980b, Knapp et al. 1998).

A manutenção de órgãos rudimentares do sexo oposto nas flores masculinas e femininas de plantas dióicas mostra que a separação dos sexos é um fenômeno relativamente recente (Rottenberg 1998). Adicionalmente, a presença ocasional de flores hermafroditas em indivíduos femininos indica que a separação dos sexos em T. guianensis é ainda incompleta, mostrando sinais de um suposto ancestral hermafrodita. Fleig (1989) mostrou que em T. guianensis e em seis outras espécies brasileiras nativas, pertencentes a três outros gêneros da família (Anacardium, Lythraea e Schinus) apresentam flores unissexuais com órgãos não funcionais do sexo oposto. Com base nestas afirmações é possível supor que a dioicia em $T$. guianensis, evoluiu a partir da bissexualidade.

Estruturas de atração das flores são mais importantes para a função masculina (doação de pólen) do que para a feminina (recepção de pólen) (Janzen 1977, Charlesworth \& Charlesworth 1987). Esta analogia pode ser feita para inflorescências de $T$. guianensis que foram consideradas como as unidades de atração na espécie. Inflorescências masculinas maiores e com maior número de flores parecem ser a regra entre plantas dióicas (Bawa \& Opler 1975, Lloyd \& Webb 1977, Beach 1981, Bullock et al. 1983, Armstrong \& Irvine 1989a). Esse fenômeno aumenta o "display" floral masculino, melhorando a atração de visitantes (Bawa \& Opler 1975, Anderson \& Symon 1989).

O extenso intervalo noturno adotado para observação da abertura das flores impede a determinação do momento exato de ocorrência da antese nesse intervalo. No entanto, durante o censo realizado às 6:00 $\mathrm{h}$, notou-se que boa parte das flores dos dois sexos estavam parcialmente abertas, sugerindo que a antese, para as duas formas florais naquele período, deve ser crepuscular. Nesse sentido, a antese das flores masculinas foi mais intensa ao amanhecer. Uma vez que flores masculinas parcialmente abertas já liberavam pólen, e que femininas neste estágio de desenvolvimento ainda não produziam néctar, pode-se afirmar que maior número de novas flores masculinas estava funcional nas primeiras horas do dia, elevando o "display" floral de indivíduos masculinos.

Por outro lado a maior longevidade das flores femininas em $T$. guianensis, deve aumentar as chances de polinização, por permitir que estas fiquem mais tempo expostas aos polinizadores. Deve ser notado que a alta longevidade das flores pistiladas é acompanhada pela manutenção na produção de néctar na maioria das flores coletadas até o oitavo dia após a antese. Maior duração das flores pistiladas também foi encontrada em Jacaratia dolichaula (Donn. Sm.) Woodson por Bawa (1980b) e em 14 das 17 espécies monóicas e dióicas estudadas por Primack (1985). Dessa maneira, em T. guianensis, enquanto as plantas masculinas investem em maior "display" floral, possivelmente para aumentar a atração de visitantes florais e a exportação de pólen, as plantas femininas possuem maior longevidade floral, que também pode ter papel similar, aumentando as chances de polinização e produção de frutos (Primack 1985).

A polinização de plantas nos trópicos por insetos generalistas com alta sensibilidade a mudanças nos recursos florais (Beach 1981), e pequeno movimento entre plantas (Givnish 1980, Anderson \& Symon 1989), pode levar a altas porcentagens de autopolinização e geitonogamia (Frankie et al. 1976, Bawa 1980a, Lloyd 1982). Esse fenômeno é ainda mais evidente em árvores que possuem grande copa e maior número de flores (Bawa 1974, Bawa \& Opler 1975, Kato \& Nagamasu 1995). Por outro lado, plantas lenhosas de modo geral são muito longevas, necessitando polinização cruzada para aumentar a variabilidade gênica de suas populações e para evitar a homozigose de genes deletérios e depressão endogâmica (Bawa 1980a, Knapp et al. 1998). Estes fatores combinados poderiam ser as principais pressões ecológicas para a evolução da dioicia em plantas tropicais (Bawa \& Opler 1975, Bawa 1980a, Bawa \& Beach 1981).

A grande riqueza de visitantes florais de T. guianensis está em acordo com a idéia de que, nos trópicos, a dioicia está associada à atração de grande diversidade de insetos generalistas e poucos especializados (Bawa \& Opler 1975, Bawa 1980a, Beach 1981). Esta idéia foi contestada e debatida em meados da década passada (Renner \& Feil 1993, Bawa 1994) e, de fato, existem alguns exemplos de espécies dióicas tropicais que são polinizadas pelo vento (Bullock 1994) ou mesmo visitadas por pequena variedade de 
insetos (Bullock et al. 1983), mas este não é o caso de T. guianensis. Devemos notar que o método adotado no presente estudo, para determinação de visitantes florais, não permite distinguir polinizadores efetivos de pilhadores de recursos, nem determinar a eficiência de polinização das espécies de visitantes florais. Informações desta natureza dependem de análises do comportamento específico dos visitantes, não realizadas até o momento para $T$. guianensis.

O desenvolvimento e a maturação de frutos decorrentes de apomixia são inexpressivos na espécie, mas a simples existência da apomixia mostra que a reprodução sexuada não é exclusiva. A apomixia facultativa pode ser um mecanismo para garantir a reprodução por sementes em situações em que ocorre deficiência nos serviços de polinização (Richards 1986) e seria interessante verificar se sua frequiência não é maior em outras populações da espécie.

A produção de frutos em T. guianensis $(23,5 \%)$ foi similar àquela apresentada por Cabralea canjerana subsp. polytricha (A. Juss.) Penn. (32\%), uma espécie dióica de cerrado com eficiente sistema de polinização (Fuzeto et al. 2001). Por outro lado, a percentagem de frutos produzidos em $T$. guianensis foi sempre maior que aquela observada para seis outras espécies dióicas de cerrado (entre 0,5\% e 9\%; Oliveira 1996). Quando comparada com a produção de frutos de espécies hermafroditas da região, como Casearia grandiflora Camb. (Machado \& Oliveira 2000) e Copaifera langsdorffi Desf. (Freitas \& Oliveira 2002), ambas fortemente auto-incompatíveis e polinizadas por insetos generalistas, observamos que a percentagem de frutos formados em $T$. guianensis é semelhante ao da primeira espécie $(32 \%)$ e muito maior que a da segunda $(0,5 \%)$. A maturação de frutos na população estudada $(12,1 \%)$ também foi alta quando comparada àquela de outras espécies dióicas entomófilas que apresentam limitação de pólen [1\% em Myristica insipida R. Br. (Armstrong \& Irvine 1989a) e em Neolitsea dealbata R. Br. (House 1992); 1,5\% em Virola sebifera Aubl. (E. Lenza \& P.E. Oliveira, dados não publicados)]. Com base nas comparações apresentadas acima, pode-se afirmar que há serviço de polinização eficiente e não ocorre limitação de pólen para a formação de frutos em $T$. guianensis.

Os resultados do presente estudo indicam que a espécie é dependente de vetores bióticos para sua reprodução sexuada e que, apesar de generalistas, tais polinizadores são eficientes na polinização de T. guianensis, mesmo em áreas antropizadas como a mata do Parque do Sabiá. Como não existe nenhuma evidência de multiplicação vegetativa, estes dados sugerem que a eficiência da reprodução sexuada deve ser suficiente para explicar a ampla distribuição e importância ecológica da espécie. Além do sucesso de formação de frutos, provavelmente outros fatores como a dispersão de propágulos e a estratégia de germinação e estabelecimento devem ser estudados para entender o sucesso reprodutivo e ecológico da espécie.

Agradecimentos - À Capes e ao CNPq pelo apoio financeiro por meio de bolsa de mestrado para o primeiro autor. Ao Departamento de Ecologia da UnB, ao Instituto de Biologia da UFU e à direção do Parque do Sabiá pelo apoio logístico durante a realização do trabalho. A Júlio Mendes, Marcelino Champanhat Boaventura e Ivone Diniz pela identificação dos insetos. A Simone Carolina pelo desenho da prancha das flores.

\section{Referências bibliográficas}

ACKERLY, D.D., RANKIN-DE-MERONA, J.M. \& RODRIGUES, W.A. 1990. Tree densities and sex ratios in breeding populations of dioecious Central Amazonian Myristicaceae. Journal of Tropical Ecology 6:239-248.

ANDERSON, G.J. \& SYMON, D.E. 1989. Functional dioecy and andromonoecy in Solanum. Evolution 43:204-219.

ARMSTRONG, J.E. \& IRVINE, A.K. 1989a. Flowering, sex ratio, pollen-ovule ratios, fruit set and reproductive effort of a dioecious tree, Myristica insipida (Myristicaceae), in two diferent rain forest communities. American Journal of Botany 76:74-85.

ARMSTRONG, J.E. \& IRVINE, A.K. 1989b. Floral biology of Myristica insipida (Myristicaceae), a distinctive beetle pollination syndrome. American Journal of Botany 76:86-94.

AUGSPURGER, C.K. 1980. Mass-flowering of a tropical shrub (Hybanthus prunifolius): influence of pollination attraction and movement. Evolution 34:475-488.

AUGSPURGER, C.K. 1981. Reproductive synchrony of a tropical plant: experimental effects of pollinators and seed predators on Hybanthus prunifolius (Violaceae). Ecology 62:775-788.

BAWA, K.S. 1974. Breeding systems of tree species of a lowland tropical community. Evolution 28:85-92.

BAWA, K.S. 1977. The reproductive biology of Cupania guatemalensis Radlk. (Sapindaceae). Evolution 31:52-63.

BAWA, K.S. 1980a. Evolution of dioecy in flowering plants. Annual Review of Ecology and Systematics 11:15-39.

BAWA, K.S. 1980b. Mimicry of male by female flowers and intrasexual competition for pollinators in Jacaratia dolichaula (D. Smith) Woodson (Caricaceae). Evolution 34:467-474.

BAWA, K.S. 1994. Pollinators of tropical dioecious angiosperms, a reassessment? No, not yet. American Journal of Botany 81:456-460.

BAWA, K.S. \& BEACH, J.H. 1981. Evolution of sexual systems in flowering plants. Annals of the Missouri Botanical Garden 68:254-274. 
BAWA, K.S. \& OPLER, P.A. 1975. Dioecism in tropical forest trees. Evolution 29:167-179.

BAWA, K.S., PERRY, D.R. \& BEACH, J.H. 1985a. Reproductive biology of tropical lowland rain forest trees. I. Sexual systems and incompatibility mechanisms. American Journal of Botany 72:331-345.

BAWA, K.S., BULLOCK, S.H., PERRY, D.R., COVILLE, R.E. \& GRAYUM, R.H. 1985b. Reproductive biology of tropical lowland rain forest trees. II. Pollination systems. American Journal of Botany 72:346-356.

BEACH, J.H. 1981. Pollinators foraging and the evolution of dioecy. The American Naturalist 118:572-577.

BULLOCK, S.H. 1985. Breeding systems in the flora of a tropical deciduous forest in Mexico. Biotropica 17:287-301.

BULLOCK, S.H. 1994. Wind pollination of neotropical dioecious trees. Biotropica 26:172-179.

BULLOCK, S.H., BEACH, J.H. \& BAWA, K.S. 1983. Episodic flowering and sexual dimorphism in Guarea rhopalocarpa in a Costa Rican rain forest. Ecology 64:851-861.

CHARLESWORTH, D. \& CHARLESWORTH, B. 1987. The effect of investiment in attractive structures and allocation to male and female functions in plants. Evolution 41:948-968.

CROAT, T.B. 1979. The sexuality of Barro Colorado Island flora (Panama). Phytologia 42:319-348.

FLEIG, M. 1989. Anacardiáceas In Flora ilustrada catarinense (R. Reitz, ed.). Herbário Barbosa Rodrigues - HBR/Empresa Catarinense de pesquisa Agropecuária, Itajaí.

FRANKIE, G., OPLER, P.A. \& BAWA, K.S. 1976. Foraging behavior of solitary bees: implications for outcrossing of a neotropical tree species. Journal of Ecology 62:881-919.

FREEMAN, D.C., DOUST, J.L., EL-KEBLAWI, A., MIGLIA, K.J. \& MCARTHUR, E.D. 1997. Sexual specialization and inbreeding avoidance in the evolution of dioecy. The Botanical Review 63:65-92.

FREITAS, C.V. \& OLIVEIRA, P.A. 2002. Biologia reprodutiva de Copaifera langsdorffii Desf. (Leguminosae, Caesalpinoideae). Revista Brasileira de Botânica 63:311-321.

FUZETO, A.P., BARBOSA, A.A.A. \& LOMÔNACO, C. 2001. Cabralea canjerana subsp. polytricha (A. Juss.) Penn. (Meliaceae), uma espécie dióica. Acta Botanica Brasilica 15:167-175.

GIVNISH, T.J. 1980. Ecological constraints in the evolution of breeding systems in seed plants: dioecy and dispersal in gymnosperms. Evolution 34:959-972.

GIVNISH, T.J. 1982. Outcrossing versus ecological constraints in the evolution of dioecy. The American Naturalist 119:849-865.
GUILHERME, F.A.G., NAKAJIMA, J.N., LIMA, C.A.P. \& VANINI, A. 1998. As fitofisionomias e a flora lenhosa nativa do Parque do Sabiá, Uberlândia, MG. Daphne 8:17-30.

HEYWOOD, V.H. 1985. Flowering plants of the world. Croom Helm, London.

HOUSE, S.M. 1992. Population density and fruit set in three dioecious tree species in Australia tropical rain forest. Journal of Ecology 80:57-69.

IBARRA-MANRÍQUEZ, G. \& OYAMA, K. 1992. Ecological correlates of reproductive traits of Mexican rain forest trees. American Journal of Botany 79:383-394.

JANZEN, D.H. 1977. A note on optimal mate selection by plants. The American Naturalist 111:365-371.

KATO, M. \& NAGAMASU, H. 1995. Dioecy in the endemic genus Dendrocacalia (Compositae) on the Bonin (Ogasawara) island. Journal of Plant Research 108:443-450.

KEARNS, C.A. \& INOUYE, D.W. 1993. Techniques for Pollination Biologists. University Press of Colorado, Niwot.

KNAPP, S., PERSSON, V. \& BLACKMORE, S. 1998. Pollen morphology and functional dioecy in Solanum (Solanaceae). Plant Systematics and Evolution 210:113-139.

LLOYD, D.G. 1982. Selection of combined versus separate sexes in seed plants. The American Naturalist 120:571-585.

LLOYD, D.G. \& WEBB, C.J. 1977. Secondary sex characters in plants. The Botanical Review 42:177-216.

MACHADO, A.O. \& OLIVEIRA, P.E. 2000. Biologia floral e reprodutiva de Casearia grandiflora Camb. (Flacourtiaceae). Revista Brasileira de Botânica 23:283-290.

MUENCHOW, G.E. \& DELESALLE, V. 1994. Pollinator response to male floral display size in two Sagittaria (Alismataceae) species. American Journal of Botany 81:568-573.

NEWSTROM, L.E., FRANKIE, G.W. \& BAKER H.G. 1994. A new classification for plant phenology based on flowering patterns in lowland tropical rain forest trees at La Selva, Costa Rica. Biotropica 26:141-159.

OLIVEIRA, P.E. 1996. Dioecy in the Cerrado vegetation of Central Brasil. Flora 191:235-243.

OLIVEIRA, P.E. \& GIBBS, P.E. 2000. Reproductive biology of woody plants in a cerrado community of the Central Brazil. Flora 195:311-329.

OLIVEIRA, P.E., GIBBS, P.E. \& BIANCHI, M. 1991. Pollination and breeding biology of Vellozia squamata (LilialesVelloziaceae): a species of the Brazilian Cerrados. Botanica Acta 104:392-398.

OLIVEIRA FILHO, A.T. \& RATTER, J.A. 1995. A study of the origin of Central Brasilian forest by the analysis of plant species distributions patterns. Edinburgh Journal of Botany 52:141-194. 
OPLER, P.A. \& BAWA, K.S. 1978. Sex ratios in tropical forest trees. Evolution 32:812-821.

PRIMACK, R.B. 1980. Phenological variation within natural populations: flowering in New Zealand montane shrubs. Journal of Ecology 68:849-862.

PRIMACK, R.B. 1985. Longevity of individual flowers. Annual Review of Ecology and Systematics 16:15-37.

PROENÇA, C.E.B. \& GIBBS, P.E. 1994. Reproductive biology of eight sympatric Myrtaceae from Central Brazil. New Phytologist 126:343-354.

RADFORD, A.E., DICKSON, W.C., MASSEY, J.R. \& BELL, C.R. 1974. Vascular Plant Systematics. Harper \& Row, New York.

RENNER, S.S. \& FEIL, J.P. 1993. Pollinators of tropical dioecious angiosperms. American Journal of Botany 81:1100-1107.

RENNER, S.S. \& RICKLEFS, R.E. 1995. Dioecy and its correlates in the flowering plants. American Journal of Botany 82:596-606.

RICHARDS, A.J. 1986. Plant breeding systems. George Allen \& Unwin, London.
ROSA, R., LIMA, S.C. \&ASSUNÇÃO, W.L. 1991. Abordagem preliminar das condições climáticas de Uberlândia. Sociedade \& Natureza 3:91-108.

ROTTENBERG, A. 1998. Sex ratio and gender stability in the dioecious plants of Israel. Botanical Journal of the Linnean Society 128:137-148.

SAKAI, A.K., WAGNER, W.L., FERGUSON, D.M. \& HERBST, D.R. 1995. Biogeographical and ecological correlates of dioecy in the Hawaiian flora. Ecology 76:2530-2543.

SILVA JÚNIOR, M.C., FELFILI, J.M., NOGUEIRA, P.E. \& REZENDE, A.V. 1998. Análise florística das mata de galeria no Distrito Federal. In Cerrado: Matas de Galeria (J.F. Ribeiro, ed.). Embrapa-CPAC, Planaltina, p.53-93.

STEPHENSON, A.G. 1982. When does outcrossing occur in a mass-flowering plant? Evolution 36:762-767.

WILLSON, M.F. 1994. Sexual selection in plants: perspectives and overview. The American Naturalist 144:13-39.

ZAR, J.H. 1999. Biostatistical Analysis, $4^{\text {th }}$ ed. Prentice Hall, Upper Saddle River. 